\title{
Editing Social Psychiatry and Psychiatric Epidemiology
}

\author{
PAUL BEBBINGTON \\ Editor, Social Psychiatry and Psychiatric Epidemiology
}

\section{BACKGROUND - THE JOURNAL}

Social Psychiatry and Psychiatric Epidemiology was founded in 1965 by some of the early giants in psychiatric epidemiology and the study of the social factors that impact on psychiatric disorder. The journal was originally called Social Psychiatry, a term that proved ambiguous and was not necessarily positively viewed, especially in the United States where it was seen as having a particular political slant. This partly motivated the expansion of the name in 1988. The purpose of the journal from its inauguration was to publish empirical research in a difficult area. It was consciously international and would publish articles in English, French or German. The increasing hegemony of English as the language of science finally ended this arrangement in 1989.

Social Psychiatry and Psychiatric Epidemiology is intended to provide a medium for the prompt publication of scientific contributions concerned with all aspects of the epidemiology of psychiatric disorders - social, biological and genetic. In addition, the journal has a particular focus on the effects of social conditions upon behaviour and the relationship between psychiatric disorders and the social environment.

Address for correspondence: Professor P. Bebbington, Royal Free and University College Medical School, UCL - Department of Psychiatry and Behavioural Sciences, Holborn Union Building, Archway Campus, Highgate Hill, London N19 5LW (United Kingdom).

Fax: +44-020-7288.3411

E-mail: P.Bebbington@ucl.ac.uk

Declaration of Interest: no funding that would constitute a conflict of interest with the content of the paper.
Contributions may be of a clinical nature provided they relate to social issues, or they may deal with specialised investigations in the fields of social psychology, sociology, anthropology, epidemiology, health service research, health economies, or public mental health. We publish papers on cross-cultural and trans-cultural themes.

The original internationalism of the journal remains. Like many journals, it has an editorial board of individuals from many countries, but in addition we have editors to represent different areas of the world: the Americas, the western Pacific, and areas of the German, Italian, Spanish and French language groups. The chief editor takes the rest. The role of the editors is in part to encourage the submission of papers from these areas.

The group of editors has been stable for many years. This is by design, as the journal has felt the advantages of continuity outweigh the disadvantages of atherosclerosis. I have been the English language group editor since 1986, and editor-in-chief since 1993. The journal is published by a small company, a subsidiary of the much larger Springer Verlag, and this permits a close relationship between the editors and the publishing team. This allows rapid response to problems and a close control of the publishing process.

\section{EDITING A PSYCHIATRIC JOURNAL IN THE $21^{\text {sT }}$ CENTURY}

This description sets the background for certain specific problems of a strategic and ethical nature that the chief editor and his colleagues must address. Thus, for us the journal is good if it serves the scientific psychiatric 
community by publishing significant articles that will be read by the target readership. However, we have the subsidiary aim of encouraging research from around the world, sometimes from areas with less in the way of a scientific tradition. In order to carry out these intentions, the journal must be attractive both to its readers and to those who publish in it. These groups overlap considerably as it is a scholarly journal serving a specialist research community, and is probably not a first port of call for most clinicians in the psychiatric professions. Most of our subscriptions are institutional.

\section{Bigger may be better}

The requirements to be attractive can be served in several ways. First, the journal has to be large enough to have a presence. Research in social psychiatry and psychiatric epidemiology, as in most areas of science, has been expanding exponentially. This means many more articles are being submitted and processed. The consequence is that the journal has moved from four issues a year, to six issues (1989), to eight issues (1997) and finally from 1998, to twelve issues. This has contributed to an appreciable reduction in the publication gap, a particular benefit to those publishing in our pages. The danger of course in increasing the yearly page count is that papers may more readily be accepted with a consequent fall in overall quality. Fortunately this has not happened - there appears to have been no drop in the rejection rate despite a greater number of papers accepted in an absolute sense.

\section{The tyranny of citation}

Despite the increase in research in the journal's topic area, it remains a specialist area, and one that until very recently has been somewhat peripheral, given the emphasis, particularly in American, on biological aspects of psychiatry. One of the drawbacks to specialism for a journal is that it makes for a smaller pool of authors likely to quote articles from it. In consequence, the more specialised journals cannot hope to achieve the highest citation standings. Every journal these days tries to maximise the citation of its articles, and this may have some adverse consequences: the encouragement of articles whose sexiness may be greater than their worth. Obviously there is a large overlap between citability and worth, but it is certainly not total. One disadvantage to a concentration on citation standings is that it reduces the willingness of editors to take risks to encourage junior researchers, or researchers from regions with a young tradition of research. Nevertheless, because of the internationalist intention of our journal, we will accept articles of a good standard that may be unlikely to be widely quoted merely because of whom or where they come from. In some cases we may even be prepared to lower the publication threshold, although not, let it be said, by much.

Internationalism does create other tensions. In particular, our commitment to publishing health service research sometimes runs counter to the interest of our international readership. Because health service research is very much placed in local contexts, it may not have obvious implications for services in other countries. This parochialism is often more apparent than real and a shift in emphasis may make clear that there is something to learn for readers even from places where service organisation is quite different. One editorial task is to point this out to authors who have failed to notice the restricted context of their work (or at least of its presentation) despite working within western industrial countries with a strong research tradition.

\section{Working with referees}

A central function of editors of scientific journals is to mediate the relationship between authors and referees in a way that advances the subject, while making all parties feel that they have been fairly and properly treated. Referees of course undertake their work as an important commitment to their discipline, and it is part of the standard economics of scientific journal production that they do not get paid for their work. Editors tend to think that this is right and good, and that those who decline on a regular basis to referee articles are not playing fair if they themselves expect to be published. Indeed, editors may sometimes feel they see too much of the seamy side of certain members of the scientific community.

It is of course the case that referees are busy people and at certain times may have to turn down refereeing duties. This causes no problem as substitutes can usually be found quickly. A minority of referees will agree to take responsibility for a paper and then completely fail to provide an assessment. This places the editor in a position like that of waiting for a bus. The decision to walk (or to obtain a further referee) is made difficult by the possibility the bus may turn up.

In our journal, we have decided to retain anonymity in the process of refereeing, although we have considered changing this, as other journals have.

\section{Working with authors}

Authors also pose problems for editors, in a number of ways. Attempts have been made by the Vancouver group to clarify these issues by setting out criteria for authorship, partly in response to certain appalling high profile 
scandals (International Committee of Medical Journal Editors, 2001). In a time when research usually needs to be large scale and has to be carried out by correspondingly large teams, the number of authors on papers has tended to increase dramatically. It sometimes appears as though the author list is longer than the meat of the paper. Large teams contain people at different stages of their careers who will have made a range of contributions to the work. Most of the task of drafting the paper will have been in the hands of two or three people. However, others may have designed the study in the first place and contributed to the grant proposal that lead to funding. Junior researchers will have mainly acted as foot soldiers, although they will have had access to drafts for comment. All of these people want a piece of the kudos that arises from publication. These days however the criteria are quite strict and largely reasonable. The Vancouver Group require that authorship should be based only on:

- substantial contribution to conception and design, or analysis and interpretation of data

- substantial contribution to drafting the article or revising it critically for important intellectual content

- according final approval of the version to be published.

All these conditions must be met (International Committee of Medical Journal Editors, 2001).

As an editor not party to the establishment of authorship requirements, I am prepared to treat them as guidelines rather that holy writ. A long author list should prompt editors (this editor) to ask the corresponding author to justify the individual inclusions. Fortunately, relatively little of the research we publish has been sponsored by drug companies, some of whom employ unacknowledged writing teams, leaving the main author as a figurehead rather than the chief executive.

Authors of course do other things that editors have to be on the lookout for. The first of these is salami slicing. Most research projects these days result in several publications and some produce a veritable army. Authors therefore have to decide on how a large project is broken down for publication. I personally think there is a place for (and I am happy to publish) a central methodological paper containing relatively few data, perhaps going no further than a detailed description of the characteristics of samples. In subsequent papers the authors can then use this as a source reference, allowing them to focus more specifically on substantive findings. All very reasonable. However, the authors of small or even medium sized projects sometimes try to puff them up by publishing a series of very short papers in different journals that could easily form fewer papers without loosing coherence and read- ability. They may do this because they feel that as many people as possible should have access to the prestige of a first authorship, or just generally to advance their standing by increasing the number of their publications. The extreme form of this is of course duplicate publishing. Authors attempt to publish what is essentially the same paper in separate outlets, after judicious variation in the title and author list. This is unlikely to occur in the very top journals (where it is glaringly obvious) but may be tried in journals of the second rung. What such authors sometimes do not realise is that editors read other journals, that they talk and write to other editors, and that the pool of referees is such that a given referee may have seen other pieces of the salami or the duplicate article. Depending on the degree of slicing or of overlap, this may lead to rejection of the current paper and a very stiff and public letter from one or more editors, or merely a request to justify the attempt at a separate publication. Salami authors also fail to grasp that the academic community may itself become perfectly aware of what they are up to. None of this is to deny the fact that secondary analysis can yield valuable information and valid papers, if done appropriately.

Double publishing is anathema to editors. However, there are types of this that I have some sympathy for. The first is where the secondary articles are written for a different (non-scientific) readership, for example in the form of a report to policy makers, or an article in a nonscientific professional magazine. In such circumstances, one would expect the style and format to be considerably different, even though some of the content would be repeated. Some research projects funded by governments carry a requirement that the governmental report should be the earliest publication. I generally have no problem with that, although there may be difficulties if material from the report is described in the popular press in a way that leaves clinicians wondering what to say to their patients about something that has not been subjected to peer review. Some journals these days apparently will not agree to publish material that has been presented at a conference. In my view this is over-scrupulous, particularly as it concerns advantage to the journal rather than to the scientific community. Likewise, I am considerate of requests for the coverage of papers in press by review articles.

\section{Conflicts of interest}

Finally, most authors have conflicts of interest, in that most research projects receive funding of some formal kind. It is probable that the greatest dangers arise from commercial sponsorship, where the belief of researchers 
that they could not be swayed by the commercial interests of the sponsors is the sort of ironic self-revelation more often to be observed in parliamentarians. However, funding by government (national or EU) departments carries the prospect of conflict of interest, particularly as such departments often insist on vetting papers before submission to scientific journals. In Britain, most government funded psychiatric research is sponsored by the Department of Health, while some falls under the Home Office. Each of these departments has an agenda that may result in (usually subtle) pressure upon researchers, and this must be the case in other polities. Even charities have axes to grind, and may not in any case be as independent as they appear. Our journal, because of its topic spread, does not publish much in the way of commercially sponsored research. For this reason, we have no policy of insisting that authors declare conflicts of interest. However, it is now apparent we should develop one.

Editors and referees also have conflicts of interest, although the structures to contain these are generally informal, relying on the good faith of the parties to acknowledge the possibility of bias. At one time, we had a policy of anonymising papers before sending them out, but our referees made it clear they could generally guess the authors' group, if not the individual authors.

The power to accept and reject papers is of course open to abuse. The best way to minimise this is to minimise the input of the editor into the decision making process. This is the purpose of relying on referees in the first place. Nevertheless, there are circumstances when I will reject a paper without referral. This is often when the paper clearly does not fall within the scope of our journal, but in some cases, the paper is of such a poor and unremediable quality that the process of refereeing would impose an unnecessary cruelty.
Where referees' views cohere, there are no grounds for adding an editorial view (the referees are after all chosen for competence). Rarely this means immediate acceptance, usually return to the authors for revision. The process of comment almost invariably improves a paper. In many cases the level of revision is easily accomplished and the paper can be accepted. In others, the authors are told clearly that further refereeing will be necessary, and that there is no guarantee of success.

A major problem arises where referees are in conflict. In these circumstances the editor has the choice of evaluating the reports and coming down on one side or another, or of sending the paper to a further referee in the hope of resolution.

Editors have the capacity to abuse their power in the matter of papers of which they are an author. One solution is for editors are never to submit papers to their own journal, but there is little evidence of such a rule being adhered to. Our journal has the advantage of several independent editors, and we have adopted the solution of having our editorial colleagues take over the whole process of review and acceptance. Given that we are a punctilious group, I have found it is often easier to place the paper elsewhere.

The position of editor involves considerable work, but is very rewarding. It conveys a real sense of assisting the progress of one's chosen discipline. As such, it is a clear privilege.

\section{REFERENCE}

International Committee of Medical Joumal Editors (2001). Uniform requirements for manuscripts submitted to biomedical journals. Retrieved October 29, 2002, from http://www.icmje.org/. 